\title{
Casting an Eye on Complexity
}

\author{
Niles Eldredge
}

Published online: 19 September 2008

(C) Springer Science + Business Media, LLC 2008

In the previous issue, I offered some thoughts on how to think about evolution (Eldredge 2008). It turns out that how "we" collectively think about evolution, starting with the most basic, fundamental conceptualization about what evolution is, has differed dramatically depending on when a given evolutionist was active, what her subdiscipline isand even to some degree on what country she was born in.

For example, a systematist or paleontologist might prefer a one-sentence definition of "evolution" as "the idea that all species that have ever lived on earth are descended from a single common ancestor." In contrast, a geneticist might prefer to define evolution as "any permanent change in genetic information." In addition, while both definitions may suffice for some systematists or geneticists, others (such as myself) would insist on adding "and the causal processes underlying such patterns of descent and/or change in genetic information."

And while there may be still other definitions of evolution, these two seem to constitute a dichotomy that has pervaded biological evolutionary theory at least since Darwin. One of them is about the origin and fates of species - and, by extension, higher taxa such as genera, families etc. The other focuses on the origin and further modification of genetically based properties of organisms (morphology, physiology, behavior). And although such nondeterministic causes of genetic change as Sewall Wright's "genetic drift" (see Eldredge 2008) are in operation, basically theories of genetic change (i.e., over and above mutational and related sorts of changes in individual organisms) are theories of adaptation

\section{N. Eldredge $(\bowtie)$}

Division of Paleontology,

American Museum of Natural History,

New York, NY, USA

e-mail: epunkeek@amnh.org through natural selection - and have been so throughout the history of evolutionary thought.

What's more, the distinction between these two fundamentally different ways of defining evolution - the "taxic" vs. the "transformationist" approach (as I initially called them in Eldredge 1979) are seldom acknowledged. The tendency (probably ever since Darwin published the Origin in 1859) is to see the "origin of species" as the simple and direct consequence of the ongoing transformation of the heritable properties of organisms via adaptation through natural selection. The thought goes: If natural selection modifies a species enough, over the course of time a new species will inevitably emerge.

But, as I will explore in detail in the next issue (dedicated to Charles Robert Darwin on the occasion of his 200th birthday), Darwin himself initially saw evolution as a "taxic" phenomenon: ancestral species give rise to descendant species, much as mothers give birth to infant children. He was thinking in these terms even on the Beagle and continued to do so after he returned home in 1836. Later, he essentially dropped this line of thought - but only after he had come up with the idea of natural selection - and switched over to thinking of evolution as essentially the simple modification of adaptations through natural selection.

Today, we have theories of "speciation"-how new species are "born" from ancestral species (see Thanukos 2008, for a useful review in these pages), and we also have theories of adaptation through natural selection. And it is clear that the two fundamentally different ways of thinking about evolution I have sketched out above are intimately related to one another: This is not an either/or dichotomy, but rather two equally valid ways of looking at evolution that resonate with one another in more of a dialectical than a dichotomous way. For though it is possible to have a descendant species arise with hardly much genetic change 
accruing between ancestor and descendant species, usually adaptive change - at the very least, in what biologist Paterson (1985) has called the "Specific Mate Recognition System" ("SMRS" for short) - lies at the heart of the derivation of a descendant species, now a separate reproductive/genetic lineage-from its ancestral species. The SMRS is the totality of reproductive adaptations that allows mates to recognize one another within sexually reproducing species. Thus, each species has its own unique SMRS, which, as a side effect, also keeps even closely related species apart from each other.

And conversely, as I have pointed out in several recent "Editor's Corners," adaptive evolution through natural selection, though in principle it can occur at any time and in any place within any clade - in practice seems to be disproportionately focused at times when new species are forming. And many speciation events seem clustered at times just after a wave of extinction has sent many species to extinction. Extinction and lineage splitting (speciation) seem to provide an extremely important context for adaptive change to occur. My experience as a paleontologist long ago taught me that most species, most of the time, are simply not accumulating genetically based adaptive change (the phenomenon of "stasis"). So I see speciation as a stimulus of adaptive change - unlike the older view that new species originate as a simple, direct byproduct of adaptive change through natural selection. There is indeed a resonance - a dialectic - set up by seeing evolution as both speciation and adaptation through natural selection. They are not the same thing, but they do resonate with one another.

Which brings us to the topic of virtually this entire issue of Evolution: Education and Outreach: the (adaptive) evolution of anatomical complexity-specifically, "the eye." For believing as I do-that all stages of evolution of a truly complex adaptation such as an arthropod, cephalopod, or vertebrate eye, presupposes a series of speciation events within a single clade - in the absence of direct knowledge of all the intermediates in that clade, a complex adaptation raises the question: How did that structure evolve? Specifically, how could such an intricate structure evolve through many stages of "less perfect" configuration to reach the final product? How can half-an-eye see, thus play a positive a role in an organism's life, to be further "perfected" as the adaptive evolutionary process wears on?

The Reverend Paley (1802), many will recall, used the complex arrangement of the many different working parts of the mechanism of a pocket watch to make a de facto anti-evolutionary analogy: Paraphrased, Paley said that just as finding a watch on the ground tells us of the necessary existence of a watchmaker, so too does the existence of complex biological structures tell us that a Designing Intelligence (for Paley, the Christian God) must have been the designer and builder. Dawkins' 1986 book The Blind Watchmaker cleverly turns this argument on its head, as he shows that the nonrandom, statistically deterministic process of natural selection is all that is needed in the way of a natural process to shape genetically based variation to further modify - and render even more intricate - complex adaptations.

But Darwin himself was worried about complex adaptations - and not just in his Origin passages on the eye. In early 1838 , in his private transmutation notebook C, p. 175, Darwin (Darwin 1987) wrote: "We never may be able to trace the steps by which the organization of the eye, passed from simpler stage to more perfect. Preserving its relations. - the wonderful power of adaptation given to organization.-This really perhaps greatest difficulty to whole theory.- "Never someone to shy away from a difficult subject, Darwin kept at it-so that by the time he wrote the Origin (1859), he "had to convince the public that complex organs could be formed in a step-by-step process....He succeeded brilliantly. Cleverly, Darwin did not try to discover a real pathway that evolution might have used to make the eye. Rather, he pointed to modern animals with different kinds of eyes (ranging from the simple to the complex) and suggested that the evolution of the human eye might have involved similar organs as intermediates."

Any guess as to who wrote these words? No, not Richard Dawkins. And no, it was not Stephen Jay Gouldor any other recognized authority on evolution. Surprise: It was Michael Behe, poster biologist of the "Intelligent Design" community, writing (p. 16) in his 1996 book Darwin's Black Box. Behe has led the charge on the "irreducible complexity" modern version of Paley's old watch argument — but even he had to admit that Darwin had a very persuasive argument when he pointed to the spectrum of simple to highly complex versions of "eyes" that still survive to the present day in various different animal groups. You will find many details along these lines in this present issue.

But if Behe threw in the towel with the gross anatomy of the eye (unlike many of his fellow creationists who still cite the eye as "irreducibly complex"), he took a step into the murkier, less charted waters of the biochemistry of photon reception-claiming that, at this level, structures and chemical processes are so complexly intermingled that they can only reflect the handiwork of an Intelligent Designer. Falsify one hoary old creationist example, they have another one for you.

But looking at the evolution of complex organ systems in a step-wise fashion-seeing a spectrum of simpler-to-morecomplex in a number of unrelated clades - does bring us back around to a taxic perspective. For the exercise is meant to fill in gaps in empirical knowledge about the details of the evolution in a single phylogenetic lineage (a "clade"). 
Evolutionary trends (see Gregory 2008) often involve examples of complexification through time - and through a series of speciation events repeated over and over through the geological history of a clade, sometimes the eye will get just a bit better-perhaps a bit more complex in one way or another. I sometimes think of the evolution of complexity as working something like a ratchet: The simple form of a structure works perfectly well given the overall niche requirements of a given species. But if a newer, betterand perhaps even more complex-form of that structure should evolve, suiting the descendant species even better to a slightly altered set of environmental circumstances, it will be conserved.

Which brings me to the most powerful connection between evolution and complexity: Today, we have a spectrum of complexity ranging from prokaryotes (Achaeans, true bacteria) up through elephants. We wonder: Which came first - the prokaryotes, or the multicellular, highly differentiated eukaryotes-i.e., bacteria or elephants? We imagine, of course, that the prokaryotes, being the simpler, must be most similar to the earliest organisms on the planet; so, assuming life began here, we predict that the oldest fossils should be prokaryotes: They are - going back over 3.5 billion years - and the only form of life on the planet for at least another 1 billion years. Then came the more complex eukaryotic microorganisms. And so on. Elephants came way later.

Thus one grand prediction of everyone's version of "evolution" is that life evolved from the simple to the complex - a prediction that at one and the same time shows the notion of evolution to be thoroughly scientific - and in this instance, abundantly corroborated.

No such equivalent predictions are forthcoming from "Intelligent Design" thinking. One would probably imagine that a truly intelligent designer would simply put the best version on the scene from the get-go, and dispense with all the simpler, less complex versions - instead of the billions of years of tinkering with what genetic information was on the scene-sometimes, though not always, coming up with something more complex. But complexity is not inevitable.
Think about it: Bacteria and other simple things are still running the planet.

I have written this essay, staring at my computer screen and occasionally glancing up to see a bright, sunshine-filled world of yellows, greens, reds, and blues in our backyard garden. I could not have done so-at least so well-only 2 weeks ago - before I had my cataract surgery. I can now see nearly perfectly out of an eye that had been badly myopic and astigmatic, since at least I was 4 years oldand had basically all but ceased to function as my UVinduced cataract worsened steadily these past few years. A plastic lens neatly inserted and popping open to take its place where my natural lens had been for so long has effected a near-miraculous change in my vision - and so in my enjoyment of life. Art imitates nature. Now that is "intelligent design."

\section{References}

Behe MJ. Darwin's black box. The biochemical challenge to evolution. New York: The Free Press; 1996.

Darwin C. Notebook C. Transcribed by D. Kohn, In Barrett PH et al. 1987. Charles Darwin's notebooks 1836-1844. Ithaca: Cornell University Press.

Darwin C. On the origin of species by means of natural selection. London: John Murray; 1859.

Dawkins R. The blind watchmaker. New York: Oxford University Press; 1986.

Eldredge N. Alternative approaches to evolutionary theory. In Schwartz $\mathrm{JH}$, Rollins HB, editors. Models and methodologies in evolutionary theory. Bull Carnegie Mus Nat Hist. 1979;13:7-19.

Eldredge, N 2008. Editor's corner: some thoughts on "adaptive peaks," "Dobzhansky's dilemma"-and how to think about evolution. Evolution: Education and Outreach. doi 10.1007/s12052-0080057-4

Gregory TR. Evolutionary trends. Evolution: Education and Outreach; 2008.

Paley W. Natural theology. London: R. Fauldner; 1802.

Paterson HEH. The recognition concept of species. In Vrba ES, editors. Species and speciation. Transvaal Mus Mongr. 1985;4:21-29.

Thanukos A. Views from understanding evolution. Parsimonious explanations punctuated patterns Evo Edu Outreach. 2008;1:138-46. doi:10.1007/s12052-007-0025-4. 